\title{
Does the second caustic ring of dark matter cause the Monoceros Ring of stars?
}

\author{
A. Natarajan ${ }^{1}$ and P. Sikivie ${ }^{1,2}$ \\ ${ }^{1}$ Department of Physics, University of Florida, Gainesville, Florida 32611, USA \\ ${ }^{2}$ Theoretical Physics Division, CERN, CH-1211 Genève 23, Switzerland
}

(Received 1 May 2007; published 10 July 2007)

\begin{abstract}
Caustic rings of dark matter were predicted to exist in the plane of the Galaxy at radii $a_{n} \simeq 40 \mathrm{kpc} / n$ for $n=1,2,3, \ldots$ The recently discovered Monoceros Ring of stars is located near the $n=2$ caustic, prompting us to consider a possible connection between these two objects. We identify two processes through which the Monoceros Ring of stars may have formed. One process is the migration of gas to an angular velocity minimum at the caustic leading to enhanced star formation there. The other is the adiabatic deformation of star orbits as the caustic slowly grows in mass and radius. The second process predicts an order $100 \%$ enhancement of the density of disk stars at the location of the caustic ring.
\end{abstract}

DOI: $10.1103 /$ PhysRevD.76.023505

PACS numbers: 95.35.+d

\section{INTRODUCTION}

The Monoceros Ring is an overdensity of stars in the plane of the Galaxy at a galactocentric distance of approximately $20 \mathrm{kpc}$. It was discovered by the Sloan Digital Sky Survey (SDSS) [1,2] and its existence was promptly confirmed by two independent collaborations [3,4]. The Monoceros Ring has been observed over 170 degrees in galactic longitude $l$ in the Galactic anticenter direction $\left(100^{\circ} \lesssim l \lesssim 270^{\circ}\right)$, and appears to be circular. Assuming it is a complete circle, the total mass in the Ring is estimated to be in the range $\left(2 \times 10^{7}\right)-\left(5 \times 10^{8}\right) M_{\odot}$ in Ref. [2], and $\left(2 \times 10^{8}\right)-\left(2 \times 10^{9}\right) M_{\odot}$ in Ref. [3]. The scale height of the Ring stars in the direction perpendicular to the Galactic plane is estimated to be $1.6 \pm 0.5 \mathrm{kpc}$ in Ref. [2], $0.75 \pm 0.04 \mathrm{kpc}$ in Ref. [3], and $1.3 \pm 0.4 \mathrm{kpc}$ in Ref. [4]. The scale height in the direction parallel to the plane is also of order kpc. The stars in the Ring move with speed approximately $220 \mathrm{~km} / \mathrm{s}$ in the direction of galactic rotation $[5,6]$. Their velocity dispersion along the line of sight is small. It was estimated to be between 20 and $30 \mathrm{~km} / \mathrm{s}$ in Ref. [2], and $20 \pm 4 \mathrm{~km} / \mathrm{s}$ in Ref. [5].

The most widely discussed interpretation of the Monoceros Ring is that it is a stream of stars resulting from the tidal disruption of a Galactic satellite [7-9]. An alternative proposal is that the Ring is a manifestation of the Galactic warp [10,11]. In this paper we explore a different proposal altogether, namely, that the Monoceros Ring of stars formed as a result of the gravitational forces exerted by the second caustic ring of dark matter in the Milky Way [12].

Caustic rings of dark matter had been predicted [13], prior to the discovery of the Monoceros Ring, to lie in the Galactic plane at radii given by the approximate law $a_{n} \simeq$ $40 \mathrm{kpc} / n$, where $n=1,2,3 \ldots$ Since the Monoceros Ring is located near the second $(n=2)$ caustic ring of dark matter, it is natural to ask whether the former is a consequence of the latter. In our proposal, the position of the Monoceros Ring in the Galactic plane and its $20 \mathrm{kpc}$ radius are immediately accounted for.
Dark matter caustics are an unavoidable consequence of the fact that cold collisionless dark matter (CDM) lies on a 3-dimensional hypersurface in 6-dimensional phase space [14-17]. The flow of dark matter in and out of the gravitational potential well of a galaxy necessarily produces caustics. These caustics are of two types, inner and outer. The inner caustics are rings when the angular momentum distribution of the dark matter is dominated by net overall rotation [18]. The singularity structure of caustic rings was discussed in detail in Ref. [15]. The ring radii were predicted in Ref. [13] using the self-similar infall model of galactic halo formation $[19,20]$, generalized to allow the dark matter particles to have nonzero angular momentum [21]. Evidence for caustic rings of dark matter distributed according to the prediction of the self-similar infall model was found in the rotation curves of exterior galaxies [22] and the rotation curve of the Milky Way [12].

Figure 1 shows the transverse section of a caustic ring. Figure 1(a) shows the flow of dark matter in the neighborhood of the caustic, whereas Fig. 1(b) shows the definition of its radius $a$ and its transverse sizes $p$ and $q$. As was mentioned already, the self-similar infall model predicts that the radius $a_{2}$ of the second caustic ring of dark matter is approximately $20 \mathrm{kpc}$ in our galaxy. The transverse sizes $p$ and $q$ are not predicted by the self-similar infall model. However, for reasons explained in Sec. II, the expectation for $p$ and $q$ is that they are of order $1 \mathrm{kpc}$ for $n=2$. So the transverse sizes of the second caustic ring of dark matter are of order the transverse sizes of the Monoceros Ring. Moreover, for $q=1 \mathrm{kpc}$, the caustic ring mass enclosed within the triangular shape of Fig. 1(b) is approximately $6 \times 10^{8} M_{\odot}$ (see Sec. II). Thus the total mass of the $n=2$ caustic ring of dark matter is of the same order of magnitude as the observed total mass of the Monoceros Ring.

One might ask whether it is possible to interpret the Monoceros Ring as a caustic in a flow of stars. Indeed it is reasonable to assume that the flow of dark matter that forms the $n=2$ caustic ring is accompanied by a flow of stars occupying the same 3-dimensional hypersurface in 


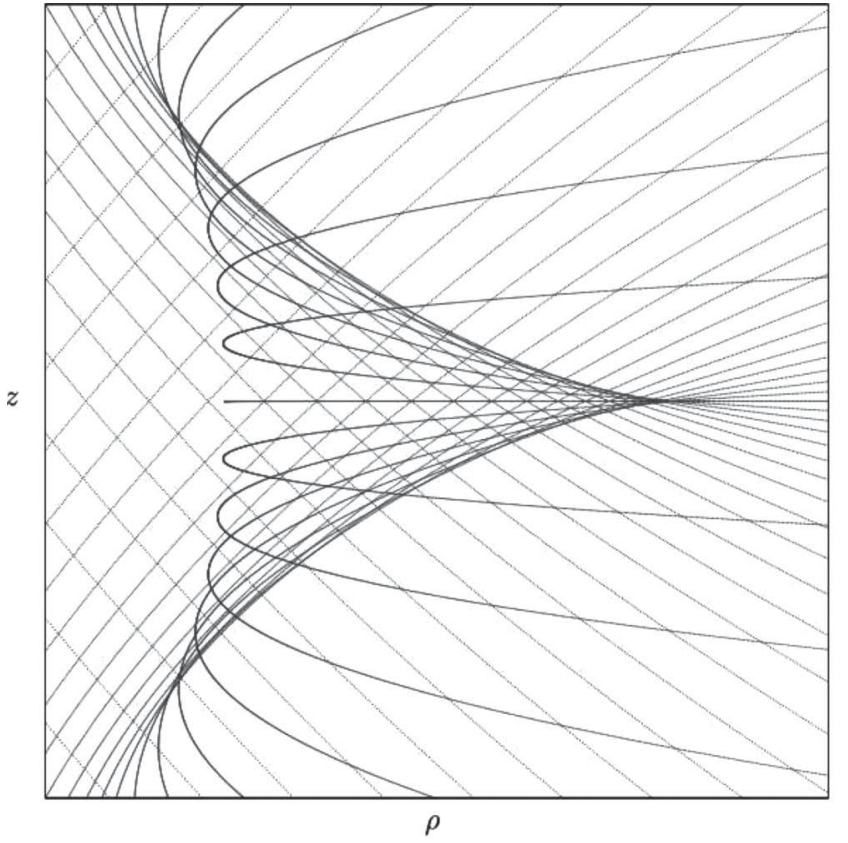

(a)

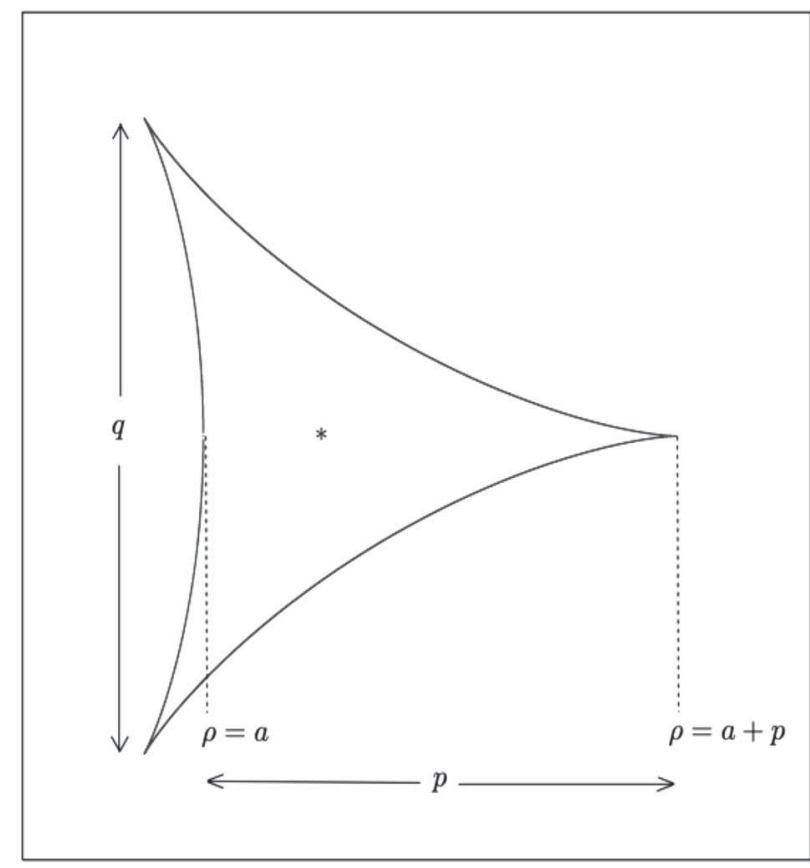

(b)

FIG. 1. (a) Dark matter trajectories forming a caustic ring of dark matter, in $\rho-z$ cross section. (b) The envelope of the trajectories shown in (a). We refer to the shape shown in (b) as the "tricusp." The figure also indicates what is meant by the radius $a$ of a caustic ring, and by its transverse sizes $p$ and $q$. The star indicates the center of the tricusp, as defined in the text.

phase space. Thus the proposal that the Monoceros Ring is a caustic in a flow of stars would explain equally well why the Monoceros Ring is in the Galactic plane and why its radius is $20 \mathrm{kpc}$. However, the proposal runs into difficulties. The first difficulty is that the self-similar infall model, which predicts the $20 \mathrm{kpc}$ radius of the $n=2$ caustic ring, also predicts that the matter in that caustic moves with a velocity of approximately $515 \mathrm{~km} / \mathrm{s}$ in the direction of Galactic rotation (see Sec. II). This is inconsistent with the $220 \mathrm{~km} / \mathrm{s}$ observed velocity of Monoceros Ring stars in the direction of galactic rotation. A second difficulty is that the matter in the caustic ring has an effective radial velocity dispersion of order $100 \mathrm{~km} / \mathrm{s}$, whereas the radial velocity dispersion of Monoceros Ring stars is only of order $20 \mathrm{~km} / \mathrm{s}$. Thus the proposal that the Monoceros Ring is a caustic of stars appears untenable. As mentioned already, the proposal we explore instead is that the Monoceros Ring is caused by the gravitational field of the $n=2$ caustic ring of dark matter.

We will find that there are two apparently viable mechanisms by which the $n=2$ caustic ring of dark matter may cause the Monoceros Ring of stars. The first mechanism is enhanced star formation at the caustic radius because viscous forces on the gas in the neighborhood of the caustic drive it towards $r=a$. The second mechanism is the adiabatic deformation of the orbits of ordinary disk stars by the caustic ring of dark matter. We will show that the increase in star density at the location of the caustic as a result of this second mechanism is of order $100 \%$.

The paper is organized as follows: In Sec. II, we give a self-contained and hopefully pedagogical description of the properties of caustic rings of dark matter, including the predictions of the self-similar infall model for the parameters that characterize the caustic rings, the observational evidence for caustic rings of dark matter, and the expected properties of the $n=2$ ring. In Sec. III, we describe the gravitational field of a caustic ring of dark matter and the perturbation it causes to the galactic rotation curve. We then analyze two mechanisms by which the second caustic ring of dark matter may cause the Monoceros Ring: the migration of gas to a sharp minimum in the angular velocity at $r=a$ implying enhanced star formation there, and the adiabatic deformation of ordinary disk star orbits by the slowly growing caustic. Section IV provides concluding remarks. Axial symmetry is assumed throughout unless stated otherwise.

\section{CAUSTIC RING PROPERTIES}

Cold collisionless dark matter particles lie on a 3dimensional hypersurface in phase space [14-16]. We refer to this 3-dimensional hypersurface as the "phase space sheet." At the location of a galactic halo, the phase space sheet is wound up so as to cover physical space multiple times. This phase space structure implies that the velocity distribution of CDM particles is everywhere discrete and that there are surfaces in physical space, called caustics, where the density of dark matter particles is very large. Discrete flows and caustics are a robust prediction of 
cold dark matter cosmology [17]. The reader may wish to consult Ref. [17] for background information and a list of references.

Galactic halos have outer caustics and inner caustics. The outer caustics are a set of simple fold $\left(A_{2}\right)$ catastrophes located on topological spheres surrounding the galaxy at radii of order hundreds of kpc. Our focus, however, is on the inner caustics, which are much closer to the galactic center. The physical shape and catastrophe structure of inner caustics depend on the angular momentum distribution of CDM particles falling onto the halo [18]. If that angular momentum distribution is dominated by net overall rotation, the inner caustics are a set of ringlike closed tubes, called "caustic rings," in or near the galactic plane. In cross section, each tube is a section of the elliptic umbilic $\left(D_{-4}\right)$ catastrophe [15].

In this section we give a detailed description of caustic rings in the limit of axial symmetry and where their crosssectional sizes, $p$ and $q$, are much smaller than their radius $a$. Under these assumptions, the distribution of CDM particles in the vicinity of the caustic ring is determined in terms of a relatively small number of parameters, which we identify. Next, we summarize the predictions of the selfsimilar infall model for the caustic ring properties. We briefly review the evidence for caustic rings of dark matter, at the radii predicted by the self-similar infall model, in the Milky Way and in other isolated spiral galaxies. Finally, we list the expected properties of the second caustic ring of dark matter in our galaxy.

\section{A. Catastrophe structure}

The caustic ring singularity was analyzed in Ref. [15] which the reader may wish to consult for details. In the limit of axial symmetry and where the transverse sizes, $p$ and $q$, of a caustic ring are much smaller than its radius $a$, the distribution of CDM in the vicinity of the caustic is given by the particle positions

$$
z(\alpha, \tau)=b \alpha \tau \quad \rho(\alpha, \tau)=a+\frac{1}{2} u\left(\tau-\tau_{0}\right)^{2}-\frac{1}{2} s \alpha^{2} .
$$

We use cylindrical coordinates $(z, \rho, \phi)$ for position in physical space. Equations (1) give particle positions at a particular time, say $t=0$. The particles are labeled by parameters $(\alpha, \tau), \alpha \equiv \frac{\pi}{2}-\theta$ where $\theta$ is the polar angle of the particle at the time of its last turnaround. $\tau$ is the time when the particle crosses the $z=0$ plane. $t-\tau$ can be thought of as the age of the particle. The particles labeled $(\alpha, \tau)$ form a circle of radius $\rho(\alpha, \tau)$ at a height $z(\alpha, \tau)$ above the $z=0$ plane.

$b, a, u, \tau_{0}$, and $s$ are constants characterizing the caustic ring. Each has physical meaning. See Ref. [15] for more precise descriptions than we give here. $a$ is the radius of the caustic ring. $\left|\tau_{0}\right|$ is of order the time a constituent particle spends in the caustic. $b$ is of order the speed of the particles in the caustic. $u$ is of order their centrifugal acceleration. $s$ characterizes the $\alpha$-dependence of specific angular momentum near the equator $(\alpha=0)$.

Figure 1 describes the caustic ring cross section. Figure 1(a) plots $(\rho(\alpha, \tau), z(\alpha, \tau))$ for continuous $\tau$, and discrete values of $\alpha$. The lines in Fig. 1(a) are the trajectories of the particles forming the flow, except that positions are plotted as a function of age, whereas for ordinary trajectories position is plotted as a function of time. Let us call the lines of Fig. 1(a) "age trajectories." Figure 1(a) shows that particle density diverges on a closed line which has the shape of an isosceles triangle, but with cusps instead of angles. We call that shape a "tricusp." The location of the tricusp is shown in Fig. 1(b) for the flow of Fig. 1(a). It is the envelope of the age trajectories. There are four flows everywhere inside the tricusp and two flows everywhere outside. The caustic, i.e. the surface where the density diverges, lies at the boundary between the region with four flows and the region with two flows.

The physical space density is given by

$$
d(\rho, z)=\left.\frac{1}{\rho} \sum_{j=1}^{N(\rho, z)} \frac{d M}{d \Omega d \tau}(\alpha, \tau) \frac{\cos \alpha}{|D(\alpha, \tau)|}\right|_{\left(\alpha_{j}(\rho, z), \tau_{j}(\rho, z)\right)},
$$

where $\alpha_{j}(\rho, z)$ and $\tau_{j}(\rho, z)$, with $j=1 \ldots N(\rho, z)$, are the solutions of $\rho(\alpha, \tau)=\rho$ and $z(\alpha, \tau)=z \cdot N(\rho, z)$ is the number of flows at position $(\rho, z)$; thus, $N=4$ inside the tricusp and $N=2$ outside. $D(\alpha, \tau)$ is the Jacobian determinant of the map $(\alpha, \tau) \rightarrow(\rho, z)$ :

$$
D(\alpha, \tau) \equiv \operatorname{det}\left(\frac{\partial(\rho, z)}{\partial(\alpha, \tau)}\right)=-b\left[u \tau\left(\tau-\tau_{0}\right)+s \alpha^{2}\right] .
$$

$\frac{d M}{d \Omega d \tau}=\frac{d M}{2 \pi \cos \alpha d \alpha d \tau}$ is the mass falling in per unit solid angle and unit time. The tricusp perimeter is the locus of points $(\rho(\alpha, \tau), z(\alpha, \tau))$ for which $D(\alpha, \tau)=0$. We call $p$ and $q$ the sizes of the tricusp in the $\rho$ and $z$ directions, respectively; see Fig. 1(b). They are given by

$$
p=\frac{1}{2} u \tau_{0}^{2}, \quad q=\frac{\sqrt{27}}{4} \frac{b}{\sqrt{u s}} p .
$$

One of the cusps of the tricusp points away from the galactic center.

Consider the 3-dimensional space spanned by the two physical coordinates $\rho$ and $z$ plus the constant $\tau_{0} . \tau_{0}$ can be positive or negative. In $\left(\rho, z, \tau_{0}\right)$ space, the tricusp is perpendicular to the $\tau_{0}$ axis with one cusp pointing in the positive $\rho$ direction. As $\tau_{0}$ varies from negative to positive values, the size of the tricusp, which varies as $\tau_{0}^{2}$, shrinks to zero and then increases again. The structure in the neighborhood of $\tau_{0}=0$ is the (full) elliptic umbilic catastrophe $\left(D_{-4}\right)$, one of the elementary catastrophes in three dimensions. Thus the tricusp, i.e. the cross section of a caustic ring, is a section of the elliptic umbilic.

If the $z$-axis is rescaled relative to the $\rho$-axis so as to make the tricusp equilateral, the tricusp has a $Z_{3}$ symmetry 
[15] consisting of rotations by multiples of $\frac{2 \pi}{3}$ about the point of coordinates $\left(\rho_{c}, z_{c}\right)=(a+p / 4,0)$. This point may thus be called the center of the tricusp. It is indicated by a star in Fig. 1(b). As an example of how the above formalism is to be used, let us derive a formula for the density $d_{c}$ at the center of the tricusp. Using Eqs. (1), one finds that the four flows there have parameter values $(\alpha, \tau)=\left(0, \frac{3}{2} \tau_{0}\right),\left(0, \frac{1}{2} \tau_{0}\right),\left(+\sqrt{\frac{3 p}{2 s}}, 0\right)$, and $\left(-\sqrt{\frac{3 p}{2 s}}, 0\right)$. The corresponding values of the Jacobian determinant are $D=$ $-\frac{3}{2} b p, \frac{1}{2} b p,-\frac{3}{2} b p$, and $-\frac{3}{2} b p$. Inserting these in Eq. (2), one finds

$$
d_{c}=\frac{4}{a b p} \frac{d M}{d \Omega d \tau} .
$$

In obtaining this result, we approximated $\rho$ by $a$ and $\cos \alpha$ by 1 in Eq. (2), and neglected the $\alpha$-dependence of $\frac{d M}{d \Omega d \tau}$. These approximations are appropriate since we assume that $p$ and $q$ are small relative to $a$. For the mass per unit length enclosed within the tricusp, we find by numerical integration

$$
\lambda=0.6 p q d_{c} .
$$

Note that some of the mass associated with the caustic ring lies outside the tricusp. In particular, when $p=q=0$, $\lambda=0$ but there is still a caustic ring and an associated overdensity. In that limit, the tricusp has collapsed to a point, and the particle density diverges as the inverse distance to that point [15].

Finally, we describe the velocities of the particles that constitute the caustic ring. Let us write the velocity of particle labeled $(\alpha, \tau)$ as $\vec{v}(\alpha, \tau)=v_{\phi}(\alpha, \tau) \hat{\phi}+$ $v_{\rho}(\alpha, \tau) \hat{\rho}+v_{z}(\alpha, \tau) \hat{z}$. For $p, q \ll a$, all the particles in the caustic have the same speed $v$ because they have come to the caustic from a small region (near the equator) of the turnaround sphere, along neighboring trajectories. The main component of velocity is in the $\hat{\phi}$ direction: $v_{\phi} \simeq$ $v$. The speed $v$ is related to the centrifugal acceleration $u$ by

$$
u=\frac{v^{2}}{a} .
$$

In the case of a stationary flow, the velocity components in the $\hat{\rho}$ and $\hat{z}$ directions are

$$
v_{\rho}=-\frac{\partial \rho}{\partial \tau}=-u\left(\tau-\tau_{0}\right), \quad v_{z}=-\frac{\partial z}{\partial \tau}=-b \alpha .
$$

Here we use the fact that, in case of stationary flow, the particle positions are functions only of their age $t-\tau$. For the four flows within the tricusp, $v_{\rho}$ and $v_{z}$ are of order $u \tau_{0}=v \sqrt{\frac{2 p}{a}}$. For the four flows at the center, for example, $\left(v_{\rho}, v_{z}\right)=\left(-\frac{1}{2} u \tau_{0}, 0\right), \quad\left(+\frac{1}{2} u \tau_{0}, 0\right), \quad\left(u \tau_{0},-\frac{1}{2} \sqrt{\frac{3}{\zeta}} u \tau_{0}\right)$, $\left(u \tau_{0},+\frac{1}{2} \sqrt{\frac{3}{\zeta}} u \tau_{0}\right)$, where $\zeta \equiv \frac{u s}{b^{2}}=\frac{27 p^{2}}{16 q^{2}}$.
Caustic rings grow in mass and radius on cosmological time scales, implying that the flow is not exactly stationary. However, provided one has information on the manner in which the growth occurs, the time dependence is easily included. For example, if the growth in size is tantamount to an increase in the caustic radius at the rate $\dot{a}$, the velocities are still as described in the previous paragraph except $v_{\rho}$ is replaced with $v_{\rho}+\dot{a}$. If the growth is tantamount to expansion in all directions by a scale factor $R(t)$, $v_{\rho}$ is replaced by $v_{\rho}+\frac{\dot{R}}{R} \rho$ and $v_{z}$ by $v_{z}+\frac{\dot{R}}{R} z$.

Thus far, we have given a detailed description of a caustic ring in the limit of axial symmetry and where the transverse sizes $p$ and $q$ of the ring are much smaller than its radius $a$. The description is in terms of a small number of parameters: $a, b, u, \tau_{0}, s$, and $\frac{d M}{d \Omega d \tau}$. We now turn to the self-similar model of galactic halo formation to obtain estimates of these parameters for the caustic rings in actual halos.

\section{B. Predictions of the self-similar infall model}

The self-similar model of galactic halo formation [1921] assumes that the entire halo phase space distribution is unchanged in time except for a rescaling of all lengths by a scale factor $R(t)$, and all velocities by $\frac{R(t)}{t}$ where $t$ is cosmic time. Physical space densities scale as $\frac{1}{t^{2}}$. A spherically symmetric overdensity in an Einstein-de Sitter universe $\left(\Omega_{\text {matter }}=1\right)$ has self-similar evolution provided its initial profile is a power law $[19,20]$

$$
\frac{\delta M_{i}}{M_{i}}=\left(\frac{M_{0}}{M_{i}}\right)^{\epsilon},
$$

where $M_{i}$ is the mass interior to initial radius $r_{i}, \delta M_{i}$ is the corresponding extra mass, and $\epsilon$ is a parameter with the $a$ priori range $0 \leq \epsilon \leq 1$. The scale factor $R(t)$ is proportional to $t^{(2 / 3)+(2 / 9 \epsilon)}$. The rotation curve is flat at small $r$ provided $0 \leq \epsilon \leq \frac{2}{3}$ [19]. In an average sense, $\epsilon$ is related to the slope of the power spectrum of density perturbations on galactic scales [23]. The standard CDM power spectrum implies $\epsilon$ is in the range 0.2 to 0.35 [21].

The accelerated expansion [24] of the universe is not consistent with Einstein-de Sitter cosmology, nor therefore with strict self-similarity. This is not a serious shortcoming of the self-similar model, however, because galactic halos were formed for the most part long before the onset of accelerated expansion, when the universe was accurately described by Einstein-de Sitter cosmology.

The original spherically symmetric self-similar model $[19,20]$ assumes radial orbits for all the particles. Whereas this approximation is reasonable when describing the outer parts of a galactic halo, it is inadequate for the inner parts. Specifically, in the approximation of radial orbits, all particles pass through the galactic center each time they fall in and out of the galaxy, causing the density to diverge at the center as $\frac{1}{r^{2}}$. The halo contribution to the rotation curve 
then approaches a constant as $r \rightarrow 0$. In actual galaxies, the central parts are dominated by baryons, and the halo contribution to the rotation curve goes to zero at the center.

However, the self-similar model can be generalized [21] to allow the dark matter particles to have angular momentum. Self-similarity is maintained provided the specific angular momentum distribution on the turnaround sphere at time $t$ is of the form

$$
\vec{\ell}(\hat{r}, t)=\vec{j}(\hat{r}) \frac{R^{2}(t)}{t},
$$

where $R(t)$ is the turnaround radius and $\vec{j}(\hat{r})$ is a dimensionless and time-independent angular momentum distribution. When angular momentum is included, particle orbits avoid the galactic center, the inner parts of the halo are depleted, and the halo contribution to the rotation curve goes to zero at $r=0$, as it should. In the Milky Way, approximately half of the rotation velocity squared at our location is due to dark matter, and half is due to baryonic matter. This determines the average magnitude $\bar{j}$ of the rescaled angular momentum distribution $\vec{j}(\hat{r})$ to be of order $\bar{j} \sim 0.2$ for the Milky Way halo [21].

The caustic rings of dark matter occur where the particles with the most angular momentum are at their distance of closest approach to the galactic center $[15,18]$. In the spherically symmetric model, the orbits are radial and hence the distance of closest approach vanishes for all particles. In that case all the caustic rings collapse to a single caustic point at the center of spherical symmetry. In the more realistic self-similar model with angular momentum, the caustic rings have finite radii. The radii are predicted in terms of the maximum value $j_{\max }$ of the dimensionless angular momentum distribution $\vec{j}(\hat{r})$ [13]:

$$
\left\{a_{n}: n=1,2,3,4,5, \ldots\right\} \simeq \frac{40 \mathrm{kpc}}{n}\left(\frac{v_{\mathrm{rot}}}{220 \mathrm{~km} / \mathrm{s}}\right)\left(\frac{j_{\max }}{0.25}\right),
$$

where $v_{\text {rot }}$ is the rotation velocity of the galaxy. Equation (11) is for the particular case $\epsilon=0.3$. However, the $a_{n} \propto \frac{1}{n}$ approximate behavior holds for all $\epsilon$ in the relevant range $0.2 \leq \epsilon \leq 0.35$, so that a change in $\epsilon$ can be compensated for, as far as the $a_{n}$ values are concerned, by a change in $j_{\max }$. We will assume $\epsilon=0.3$ henceforth. The self-similar model does not predict $j_{\max }$ and allows a different $j_{\max }$ value for each galactic halo.

The relationship between $\bar{j}$ and $j_{\max }$ depends, of course, on the $\vec{j}(\hat{r})$ distribution. Let us assume the simplest distribution consistent with net overall rotation and axial symmetry: $\vec{j}(\hat{r})=j_{\max } \hat{\phi} \cos \alpha$, i.e. that the turnaround sphere is initially rigidly rotating. In that case, $\bar{j}=\frac{\pi}{4} j_{\max }$. Thus, for the Milky Way halo, the estimate $\bar{j} \sim 0.2$ implies $j_{\max } \sim$ 0.25 .

In summary so far, the self-similar infall model with axial symmetry and net overall rotation predicts caustic rings in the galactic plane at the radii specified in Eq. (11).
It also predicts the speeds $v_{n}$ of the dark matter particles forming the caustic rings and the infall rates $\left.\frac{d M}{d \Omega d \tau}\right|_{n}$. The $v_{n}$ are approximately $n$-independent:

$$
v_{n} \simeq 515 \frac{\mathrm{km}}{\mathrm{s}}\left(\frac{v_{\text {rot }}}{220 \mathrm{~km} / \mathrm{s}}\right) .
$$

The infall rates are given by

$$
\left.\frac{d M}{d \Omega d \tau} \frac{1}{v}\right|_{n}=f_{n} \frac{v_{\mathrm{rot}}^{2}}{4 \pi G}
$$

with

$$
\begin{aligned}
\left\{f_{n}: n\right. & =1,2,3,4,5, \ldots\} \\
& \simeq(11,4.6,2.9,2.1,1.7, \ldots) \times 10^{-2} .
\end{aligned}
$$

Equations (13) and (14) provide the prefactor which appears in the formulas for the density [Eqs. (2) and (5)], up to the ratio $\frac{v}{b}$, which is of order one but which may differ from one by a factor two or so. The transverse sizes, $p$ and $q$, of the caustic rings and the ratio $\frac{v}{b}$ depend on relatively more subtle properties of the velocity distribution at last turnaround [15], and are not predicted by the self-similar infall model.

Observational evidence has been found in support of Eq. (11) in the Milky Way and other spiral galaxies. We briefly describe this evidence now.

\section{Summary of observational evidence}

Caustic rings of dark matter in or near the galactic plane cause bumps in the galactic rotation curve. In Ref. [22] a set of 32 extended and well-measured rotation curves was analyzed to test the hypothesis that some of their bumps are caused by caustic rings of dark matter at the radii given by Eq. (11). For each rotation curve, the radial coordinate $r$ was rescaled according to

$$
r \rightarrow \tilde{r}=r\left(\frac{220 \mathrm{~km} / \mathrm{s}}{v_{\mathrm{rot}}}\right),
$$

where $v_{\text {rot }}$ is the rotation velocity implied by the curve. The rotation curves were then co-added. The combined rotation curve shows peaks at $\tilde{r} \simeq 20$ and $40 \mathrm{kpc}$, with a significance of $2.6 \sigma$ and $3.0 \sigma$, respectively. The result suggests not only the existence of caustic rings of dark matter at the radii given by Eq. (11), but also that the distribution of $j_{\max }$ values is peaked near $j_{\max } \simeq 0.27$.

The Milky Way rotation curve has a series of sharp rises at radii which agree at the $3 \%$ level [12] with the caustic ring radii given in Eq. (11). The inner North Galactic rotation curve of Ref. [25] has ten rises between $r=3$ and $8.5 \mathrm{kpc}$, which may be identified with caustic rings $n=$ $5,6 \ldots 14$. The rises are "sharp" in the sense that they start and end with discontinuities (kinks) in the slope of the rotation curve. Kinks in the rotation curve are predicted by caustic rings because the dark matter density diverges at the caustic. The outer Milky Way rotation curve is much 
less well measured. Nonetheless, it has a prominent rise near $r=13 \mathrm{kpc}$ which may be identified with the $n=3$ caustic ring. Finally, the IRAS map of the Galactic plane in the direction of galactic coordinates $(l, b)=\left(80^{\circ}, 0^{\circ}\right)$ has a triangular feature whose position and appearance is consistent with the imprint of the caustic ring of dark matter nearest to us ( $n=5)$ upon the gas and dust in the disk.

\section{Expectations for the $\boldsymbol{n}=\mathbf{2}$ caustic ring}

Equation (11) predicts that the $n=2$ caustic ring has radius $a_{2} \simeq 20 \mathrm{kpc}$. Equation (12) predicts that the speed of the particles constituting the ring is approximately $515 \mathrm{~km} / \mathrm{s}$. The transverse sizes $p_{2}$ and $q_{2}$ are not predicted. However, it is reasonable to expect that the $n=2$ caustic ring has properties similar to caustic rings $n=3$ and $n=5$ to 14 , for which we have the observational evidence mentioned in the previous subsection. The $p$ values of those rings can be read off from the widths of the corresponding rises in the Milky Way rotation curve [12]. For those "observed" rings, one finds that $p / a$ ranges from 0.015 to 0.1 , with an average value of 0.05 . We thus expect $p_{2}$ to be of order $1 \mathrm{kpc}$, and very likely between 0.3 and $2 \mathrm{kpc}$. The rises in the rotation curve do not inform us about the values of $q$. However, the triangular feature in the IRAS map provides values of both $p$ and $q$ for the $n=5$ caustic ring. In that case $\frac{q}{p} \simeq 1.5$. We therefore expect $q_{2}$ to be of order $1 \mathrm{kpc}$ as well.

The prediction for the dark matter density at the central point of the tricusp is obtained by combining Eqs. (5), (13), and (14):

$$
d_{c, 2}=8 \times 10^{-3} \frac{M_{\odot}}{\mathrm{pc}^{3}}\left(\frac{v_{2}}{b_{2}}\right)\left(\frac{\mathrm{kpc}}{p_{2}}\right) .
$$

Using Eq. (6), we obtain the predicted dark matter mass per unit length enclosed within the tricusp

$$
\lambda_{2}=5 \times 10^{6} \frac{M_{\odot}}{\mathrm{kpc}}\left(\frac{v_{2}}{b_{2}}\right)\left(\frac{q_{2}}{\mathrm{kpc}}\right) .
$$

Since $b_{2}$ is of order $v_{2}$, the total mass $2 \pi a_{2} \lambda_{2}$ enclosed within the tricusp is of order $6 \times 10^{8} M_{\odot}$. Finally, from Eq. (8) we learn that the transverse velocity components $v_{\rho}$ and $v_{z}$ of the particles within the tricusp are of order $(515 \mathrm{~km} / \mathrm{s}) \sqrt{\frac{2 p p_{2}}{a_{2}}} \sim 160 \mathrm{~km} / \mathrm{s}$.

\section{EFFECT OF A CAUSTIC RING OF DARK MATTER UPON BARYONIC MATTER IN THE DISK}

In this section, we first describe the gravitational field of a caustic ring of dark matter and its effect on the Galactic rotation curve, assuming that the caustic ring lies in the plane of the disk. Next we identify two possible mechanisms by which a ring of stars may form due to the presence of a caustic ring of dark matter. The first mechanism is the migration of gas to the caustic ring radius, leading to enhanced star formation there. The second is the adiabatic deformation of star orbits as the caustic ring slowly grows in size.

\section{A. Gravitational field of a caustic ring}

As before, we assume that the transverse dimensions, $p$ and $q$, of the caustic ring are small compared to its radius $a$. In that limit, when calculating the gravitational field $\vec{g}$ of the caustic ring at a distance of order $p$ or $q$ from the ring, we may neglect its curvature and pretend that it is a straight tube. Then

$$
\vec{g}_{c}(\rho, z)=-2 G \int d \rho^{\prime} d z^{\prime} d\left(\rho^{\prime}, z^{\prime}\right) \frac{\left(\rho-\rho^{\prime}, z-z^{\prime}\right)}{\left(\rho-\rho^{\prime}\right)^{2}+\left(z-z^{\prime}\right)^{2}} .
$$

Using Eq. (2), changing variables $\left(\rho^{\prime}, z^{\prime}\right) \rightarrow(\alpha, \tau)$, neglecting the $(\alpha, \tau)$ dependence of $\frac{d M}{d \Omega d \tau}(\alpha, \tau)$ over the size of the caustic, and approximating $\cos \alpha \simeq 1$, we obtain

$$
\begin{aligned}
\vec{g}_{c}(\rho, z)= & -\frac{2 G}{\rho} \frac{d M}{d \Omega d \tau} \\
& \times \int d \alpha d \tau \frac{(\rho-\rho(\alpha, \tau), z-z(\alpha, \tau))}{(\rho-\rho(\alpha, \tau))^{2}+(z-z(\alpha, \tau))^{2}} .
\end{aligned}
$$

Equations (1) provide the functions $\rho(\alpha, \tau)$ and $z(\alpha, \tau)$. The integral on the right-hand side (rhs) of Eq. (19) may be evaluated numerically for all $\rho$ and $z$. In the galactic plane

$$
\vec{g}_{c}(r, 0)=-\frac{8 \pi G}{r b} \frac{d M}{d \Omega d \tau} I\left(\zeta, \frac{r-a}{p}\right) \hat{r}
$$

with $\zeta=\frac{s u}{b^{2}}$ (as before) and

$$
I(\zeta, X) \equiv \frac{1}{2 \pi} \int d A d T \frac{X-(T-1)^{2}+\zeta A^{2}}{\left[X-(T-1)^{2}+\zeta A^{2}\right]^{2}+4 A^{2} T^{2}},
$$

where $X=\frac{r-a}{p}$. For $\zeta=1$ one can do the integral by analytical methods, with the result

$$
\begin{aligned}
I(1, X) & =-\frac{1}{2} \quad \text { for } X<0 \\
& =-\frac{1}{2}+\sqrt{X} \text { for } 0<X<1 \\
& =+\frac{1}{2} \text { for } X>1 .
\end{aligned}
$$

A plot of $I(1, X)$ is shown in Fig. 2(a). $I(1, X)$ has an upward kink at $X=0$ and a downward kink at $X=1$. The kinks are due to the divergent behavior of the dark matter density in the $z=0$ plane at $r=a$ and $r=a+p$. The shape of $I(\zeta, X)$ with $\zeta \neq 1$ is similar to that of $I(1, X)$. The amount $\Delta I(\zeta)$ by which $I(\zeta, X)$ rises between $X=0$ and $X=1$ depends on $\zeta$. A plot of $\Delta I(\zeta)$ is given in Ref. [15]. $\Delta I(\zeta)$ is near one for $\zeta$ of order one, and $\Delta I(1)=$ 1. 


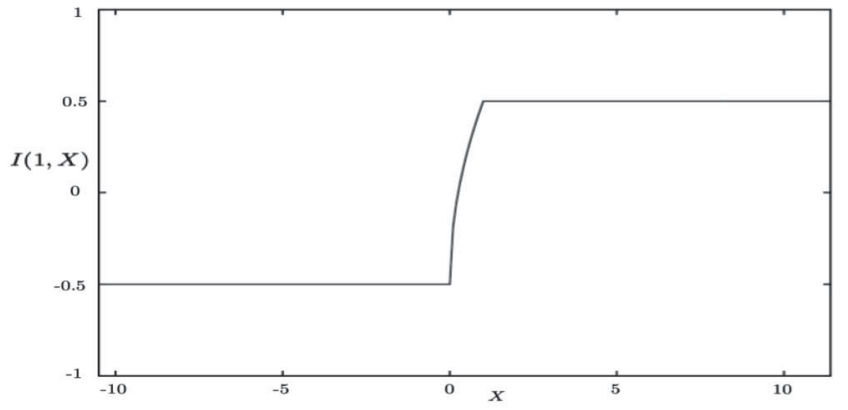

(a)

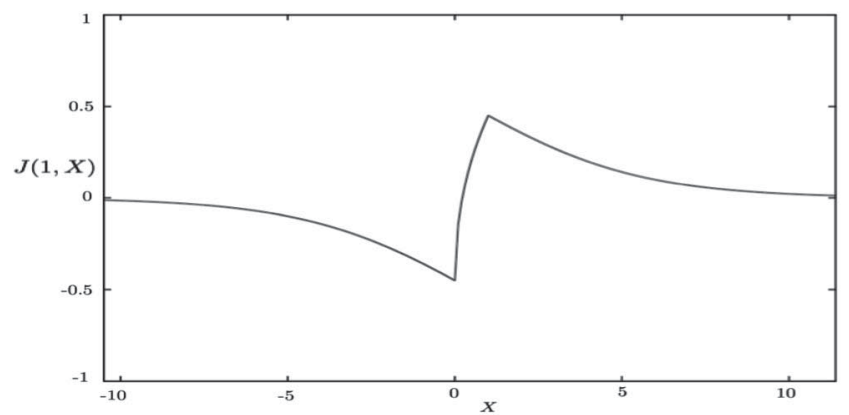

(b)

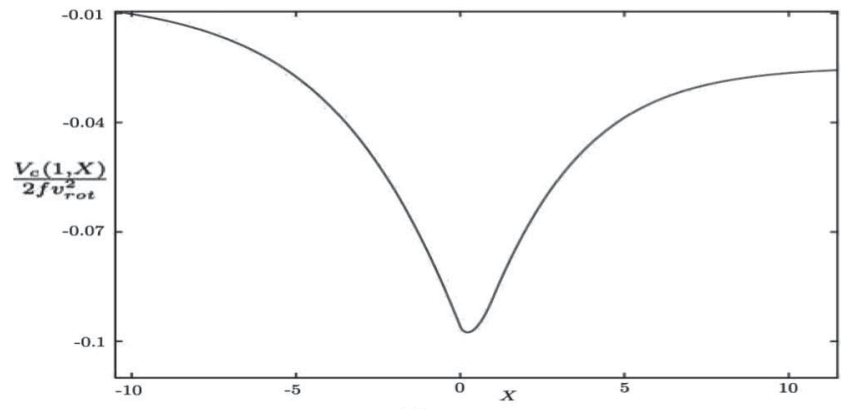

(c)

FIG. 2. (a) The function $I(1, X)$ defined in Eq. (22), with $X \equiv$ $\frac{r-a}{p}$. (b) The function $J(X)$ defined in Eq. (26) for $v=b, \zeta=1$, and $H(X)=-\frac{1}{2} \tanh \left(\frac{X}{5}\right)$. (c) The function $V_{c}(X)$ defined in Eq. (48), in units of $2 f v_{\text {rot }}^{2}$, for $J(X)$ as in (b).

Substituting Eq. (13) into Eq. (20), we obtain

$$
\vec{g}_{c}(r, 0)=-2 f \frac{v_{\text {rot }}^{2}}{r} \frac{v}{b} I\left(\zeta, \frac{r-a}{p}\right) \hat{r} .
$$

Since $\frac{v_{\text {rot }}^{2}}{r}$ is the gravitational field of the galaxy as a whole, and $\frac{v}{b}$ and $I\left(\zeta, \frac{r-a}{p}\right)$ are both of order one whereas $f$ is of order a few percent, Eq. (23) implies that the caustic ring of dark matter causes only a small perturbation on the local gravitational field.

Consider a smooth halo which produces a flat rotation curve with rotation velocity $v_{\text {rot }}$. If we add to the halo a caustic ring of dark matter of radius $a$, the gravitational field near $r=a$ would be $\vec{g}(\vec{r})=-g(r) \hat{r}$ with

$$
g(r)=\frac{v_{\mathrm{rot}}^{2}}{r}\left[1+2 f \frac{v}{b} I\left(\zeta, \frac{r-a}{p}\right)\right] .
$$

However, this is not what we want to do. Indeed, the caustic ring of dark matter is not an addition to the smooth halo. Rather, it is the outcome of redistributing dark matter already present in the halo. One can see that it is incorrect merely to add caustic rings of dark matter to a smooth halo by noting that this would cause the rotation curve to rise. The rotation velocity squared would rise by the relative amount $2 f_{n} \frac{v_{n}}{b_{n}} \Delta I\left(\zeta_{n}\right)$ at the $n$th ring, for each ring. Instead, the redistribution of dark matter into caustic rings should be such that the rotation curve remains flat on average. The gravitational field in the vicinity of a caustic ring is therefore of the form

$$
g(r)=\frac{v_{\mathrm{rot}}^{2}}{r}\left(1+2 f \frac{v}{b}\left[I\left(\zeta, \frac{r-a}{p}\right)+H(r)\right]\right),
$$

where $H(r)$ is a smooth function which steps down by the amount $\Delta I(\zeta)$ as $r$ increases from $r \ll a$ to $r \gg a$; for example, $H(r)=-\frac{1}{2} \Delta I(\zeta) \tanh \left(\frac{r-a}{p^{\prime}}\right) \cdot p^{\prime}$ is expected to be of order $a$. Let us define

$$
J(r-a) \equiv \frac{v}{b}\left[I\left(\zeta, \frac{r-a}{p}\right)+H(r)\right] .
$$

The gravitational field in the galactic plane near a caustic ring of dark matter is then

$$
g(r)=\frac{v_{\mathrm{rot}}^{2}}{r}[1+2 f J(r-a)] .
$$

Figure 2(b) shows what the function $J(r-a)$ looks like qualitatively.

\section{B. Effect on gas}

Gas in circular orbits in the galactic plane moves with angular velocity

$$
\Omega(r)=\sqrt{\frac{g(r)}{r}} .
$$

Unless $\Omega$ is $r$-independent (rigid rotation) there is shear in the velocity field. The viscous forces which result from this shear cause radial motion of the gas, as discussed by Lynden-Bell and Pringle [26]. For the sake of completeness, we repeat here relevant considerations from Ref. [26].

Consider a cylinder of radius $r$. The viscous force per unit area across the cylinder is $\rho \nu r \frac{d \Omega}{d r}$ where $\rho$ is the gas density and $\nu$ its viscosity. Hence, the viscous torque across the cylinder is

$$
\tau=2 \pi r \int d z\left(\rho \nu r \frac{d \Omega}{d r}\right) r=2 \pi \nu \sigma r^{3} \frac{d \Omega}{d r},
$$

where $\sigma=\int d z \rho$ is the mass per unit surface of the gas. Consider an annulus of width $\delta r$. Its mass is $\delta m=$ $2 \pi r \delta r \sigma$. The torque on the annulus is 


$$
\tau(r+\delta r)-\tau(r)=\delta r \frac{d}{d r}\left(2 \pi \nu \sigma r^{3} \frac{d \Omega}{d r}\right)=\frac{d}{d t} \delta L,
$$

where $\delta L=\delta m \Omega(r) r^{2}$ is its angular momentum, and $\frac{d}{d t}=$ $\frac{\partial}{\partial t}+v_{r} \frac{\partial}{\partial r}$ is the time derivative "following the motion." We have $\frac{d \delta m}{d t}=0$. Also, $\frac{\partial}{\partial t}\left[\Omega(r) r^{2}\right]=0$ because the gravitational field is determined by the mass distribution of the Galaxy as a whole and only negligibly altered by the motion of the gas. We thus obtain

$$
v_{r}=\frac{1}{\sigma r} \frac{\frac{d}{d r}\left(\nu \sigma r^{3} \frac{d \Omega}{d r}\right)}{\frac{d}{d r}\left(\Omega r^{2}\right)}
$$

for the radial velocity of the gas. The gas obeys the continuity equation

$$
\frac{\partial \sigma}{\partial t}+\frac{1}{r} \frac{\partial}{\partial r}\left(r v_{r} \sigma\right)=0 .
$$

Equation (31) neglects the backreaction pressure from the accumulation of gas at particular radii.

Let us assume that the gas is distributed uniformly $\left(\frac{d \sigma}{d r}=\right.$ $\left.\frac{d \nu}{d r}=0\right)$ to start with, and use Eq. (31) to determine in which direction it is driven by the viscous forces. In a smooth halo with flat rotation curve, $\Omega=\frac{v_{\text {rot }}}{r}$ and hence $v_{r}=-\frac{\nu}{r}$. If a caustic ring of dark matter lies in the disk, we have instead in the neighborhood of the ring

$$
\begin{aligned}
\Omega(r) & =\frac{v_{\mathrm{rot}}}{r}[1+2 f J(r-a)]^{1 / 2} \\
& =\frac{v_{\mathrm{rot}}}{r}\left[1+f J(r-a)+0\left(f^{2}\right)\right] .
\end{aligned}
$$

Inserting Eq. (33) into Eq. (31), setting $\frac{d \sigma}{d r}=\frac{d \nu}{d r}=0$, and neglecting terms of order $f^{2}$, we obtain

$$
v_{r}=-\frac{\nu}{r} \frac{1+f J(r-a)-r f \frac{d J}{d r}-r^{2} f \frac{d^{2} J}{d r^{2}}}{1+f J(r-a)+r f \frac{d J}{d r}} .
$$

To obtain the qualitative behavior, we neglect $f J(r-a)$, $r f \frac{d H}{d r}$, and $r^{2} f \frac{d^{2} H}{d r^{2}}$ vs 1 , because such terms are all of order $f$. Then

$$
v_{r} \simeq-\frac{\nu}{r} \frac{1-f r \frac{d I}{d r}-f r^{2} \frac{d^{2} I}{d r^{2}}}{1+f r \frac{d I}{d r}} .
$$

For $r<a$ and $r>a+p, \frac{d I}{d r}=0$ and hence $v_{r}=-\frac{\nu}{r}$ as in the absence of caustic. For $a<r<a+p$,

$$
v_{r} \simeq-\frac{\nu}{a} \frac{1-\frac{f a}{2 p} \frac{1}{\sqrt{X}}+\frac{f a^{2}}{4 p^{2}} \frac{1}{X^{3 / 2}}}{1+\frac{f a}{2 p} \frac{1}{\sqrt{X}}},
$$

where $X=\frac{r-a}{p}$, as before. Since $\frac{f a}{2 p}=0(1)$ whereas $\frac{f a^{2}}{4 p^{2}}=$ $0(10)$, the numerator on the rhs of Eq. (36) is always dominated by the last term. Hence,

$$
v_{r} \simeq-\frac{\nu}{a} \frac{f a^{2}}{4 p^{2}} \frac{1}{X^{3 / 2}+\frac{f a}{2 p} X} .
$$

Equation (37) shows that, within the tricusp, the gas velocity is also inward but much larger, by a factor three or more, than outside the tricusp. It increases rapidly as $r \rightarrow$ $a_{+}$. Therefore the viscous forces tend to drive the gas towards $r=a$. This happens on gas dynamic time scales which are much shorter than the cosmological time scales over which the caustic rings migrate. The accumulation of gas at the caustic ring radius may lead to enhanced star formation there. Such processes may be at work in the case of the Monoceros Ring. To the extent that such processes dominate the formation of the Monoceros Ring, the stars in the Ring should be younger than average. Reference [27] presents evidence that Monoceros Ring stars are on average bluer and therefore younger than ordinary disk stars.

\section{Effect on star orbits}

In this subsection, we consider the adiabatic deformation of disk star orbits by the slowly growing caustic ring of dark matter.

\section{Collision rate}

Adiabatic deformation of star orbits presupposes that the star collision rate is small. The time scale over which star orbits are significantly modified through gravitational scattering with other stars in a population is [28]

$$
t_{\text {relax }} \simeq 0.3 \frac{\sigma^{3}}{G^{2} m^{2} n \ln \Lambda},
$$

where $n$ is the density of stars in the population, $m$ their typical mass, $\sigma$ their velocity dispersion, and $\ln \Lambda \simeq 20$. For the stars in the Monoceros Ring, $t_{\text {relax }} \sim 10^{16} \mathrm{yr}$ if we set $m \sim M_{\odot}, \sigma \sim 20 \mathrm{~km} / \mathrm{s}$, and $n \sim 10^{-3} / \mathrm{pc}^{3}$. Since $t_{\text {relax }}$ is much greater than the age of the universe, we are justified in neglecting collisions among stars in the Ring.

\section{Orbit stability}

In a gravitational field $\vec{g}(\vec{r})=-g(r) \hat{r}$, the angular frequency squared of small radial oscillations about a circular orbit of radius $r$ is

$$
\omega^{2}(r)=\frac{1}{r^{3}} \frac{d}{d r}\left(r^{3} g(r)\right) .
$$

The orbit is stable if $\omega^{2}>0$. In the neighborhood of a caustic ring of dark matter, where the gravitational field is as given in Eq. (27), we have

$$
\omega^{2}(r)=2\left(\frac{v_{\mathrm{rot}}}{r}\right)^{2}\left(1+2 f J(r-a)+r f \frac{d J}{d r}\right) .
$$

The sum of the first two terms in the parentheses on the rhs of Eq. (40) is of order one. The third term is

$$
r f \frac{v}{b}\left(\frac{d I}{d r}+\frac{d H}{d r}\right)
$$

$\frac{d I}{d r}$ is everywhere positive, whereas $\frac{d H}{d r}$ has the qualitative 
form

$$
\frac{d H}{d r} \sim-\frac{1}{2 p^{\prime}} \frac{1}{\cosh ^{2}\left(\frac{r-a}{p^{\prime}}\right)}
$$

Since we expect $p^{\prime}$ to be of order $a$ and at any rate much larger than $f a \sim \frac{1}{20} a$, we find that circular orbits are stable everywhere in the neighborhood of a circular caustic ring of dark matter. The conclusion is valid only if the caustic ring is circular, because lack of axial symmetry may drive an instability through the phenomenon of Lindblad resonance, as we now discuss.

A particle on an orbit of radius $r$ is subjected to a radial time-dependent, but periodic, gravitational force from a noncircular caustic. The period $T=\frac{2 \pi}{\Omega} \simeq 2 \pi \frac{r}{v_{\text {rot }}}$ equals the time to go around the Galaxy once. The equation of motion for the radial coordinate of the particle is that of a harmonic oscillator of proper frequency $\omega(r)$ driven by a periodic external force with frequencies $m \Omega(r) \simeq m \frac{v_{\text {rot }}}{r}$, where $m=1,2,3, \ldots$ Resonance occurs when $\omega(r)=m \Omega(r)$. For the corresponding radii, circular orbits are unstable. Using Eqs. (33) and (40), we find the instability condition

$$
r f \frac{d J}{d r}=\left(\frac{m^{2}}{2}-1\right)[1+2 f J(r-a)]
$$

in the neighborhood of a caustic ring. Since $2 f J(r) \ll 1$, and

$$
\frac{d J}{d r} \simeq \frac{d I}{d r} \simeq \frac{1}{2} \frac{1}{\sqrt{p(\bar{r}-\bar{a})}} \Theta(r-a) \Theta(a+p-r),
$$

the instability occurs at radii

$$
r_{m} \simeq a+f^{2} \frac{a^{2}}{p} \frac{1}{\left(m^{2}-2\right)^{2}}
$$

for $m=2,3,4, \ldots$ There is no resonance for $m=1$. For $a=20 \mathrm{kpc}$ and $f=0.046$, the instabilities occur at

$$
\left\{r_{m}-a: m=2,3, \ldots\right\}=(210,17, \ldots) \mathrm{pc} \frac{\mathrm{kpc}}{p} \text {. }
$$

Let us caution that these estimates take account only of the gravitational force exerted by the caustic ring of dark matter itself, and neglect the gravity of the baryons that have aggregated at the caustic.

\section{Circular orbits}

In the absence of the caustic, the effective potential for radial motion is

$$
V_{\text {eff, } 0}(r)=v_{\text {rot }}^{2} \ln r+\frac{l^{2}}{2 r^{2}},
$$

where $l$ is the specific angular momentum of the star. When the caustic is present, the effective potential is $V_{\text {eff }}(r)=$ $V_{\text {eff, } 0}(r)+V_{c}(r)$, where

$$
V_{c}(r)=2 f v_{\text {rot }}^{2} \int \frac{d r}{r} J(r-a) .
$$

$V_{c}(r)$ is plotted in Fig. 2(c) for

$$
J(r-a)=I\left(1, \frac{r-a}{p}\right)-\frac{1}{2} \tanh \left(\frac{r-a}{p^{\prime}}\right)
$$

with $a=20 \mathrm{kpc}, p=1 \mathrm{kpc}$, and $p^{\prime}=5 \mathrm{kpc}$. Figure 2(c) illustrates the fact that the effective potential is smooth even though its second derivative diverges at $r=a$ and $r=a+p$.

The caustic ring radius increases with time. According to the self-similar infall model, $a \propto t^{(2 / 3)+(2 / 9 \epsilon)}$. Consider a particle of specific angular momentum $l$. In the absence of the caustic, it is on a circular orbit of radius $r$ given by

$$
l^{2}=r^{3} g(r)=r^{2} v_{\text {rot }}^{2},
$$

or it is oscillating about a circular orbit of that radius. In the presence of a caustic of radius $a$, the particle is on or oscillating about a circular orbit of radius $r^{\prime}(r, a)$ given by

$$
l^{2}=r^{\prime 3} g^{\prime}\left(r^{\prime}\right)=r^{\prime 2} v_{\text {rot }}^{2}\left[1+2 f J\left(r^{\prime}-a\right)\right] .
$$

Angular momentum conservation implies

$$
r^{2}=r^{\prime}(r, a)^{2}\left[1+2 f J\left(r^{\prime}(r, a)-a\right)\right] .
$$

Figure 3 shows $r^{\prime}(r, a)$ as a function of $a$. Each line in that figure corresponds to a different value of $r$. Let $\rho(r)$ be the density of stars in the absence of the caustic, and $\rho^{\prime}(r, a)$ their density in the presence of a caustic with radius $a$.

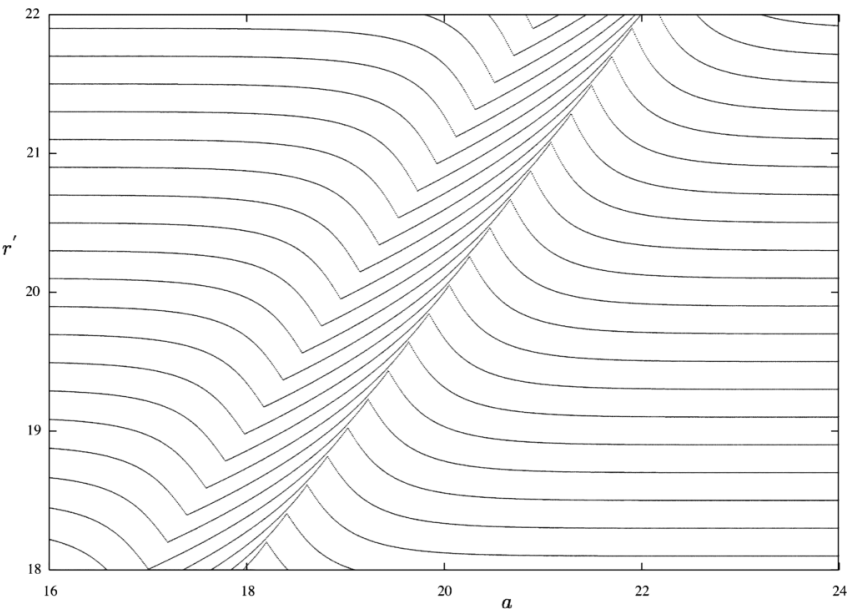

FIG. 3. The radii $r^{\prime}(r, a)$ of circular orbits in the presence of a caustic, as a function of the caustic radius $a . r$ is the radius of the orbit in the absence of caustic. Each line corresponds to a different value of $r$. Each line has an upward kink at $r^{\prime}=a+$ $p$ and a downward kink at $r^{\prime}=a$. As $a$ increases with $p$ fixed, $r^{\prime}$ first decreases from its initial value $r$ until $r^{\prime}=a+p$. For $a<$ $r^{\prime}<a+p$, i.e. when the orbit is within the tricusp, $r^{\prime}$ increases with $a$ but not as fast as $a$, until $a=r^{\prime}$. As $a$ increases yet further $r^{\prime}(r, a)$ returns to the value $r$ from which it started. Note that the radii of circular orbits pile up at $r=a$. Stars on approximately circular orbits oscillate about $r^{\prime}(r, a)$. 
Assuming that all stars are and remain on circular orbits, conservation of the number of stars implies

$$
r^{\prime} d r^{\prime} \rho^{\prime}\left(r^{\prime}, a\right)=r d r \rho(r)
$$

with $r^{\prime}(r, a)$ given by Eq. (52). One readily finds

$$
\rho^{\prime}\left(r^{\prime}, a\right)=\rho(r)\left[1+2 f J\left(r^{\prime}-a\right)+f r^{\prime} \frac{d J}{d r}\left(r^{\prime}\right)\right] .
$$

Assuming that the initial star density has no significant structure of its own, we have

$$
\rho^{\prime}(r, a)=\rho\left[1+2 f J(r-a)+f r \frac{d J}{d r}(r)\right],
$$

where $\rho$ is the initial density. The second term in brackets is an order $10 \%$ modulation of the initial density. The third term is of order $f$, like the second term, but diverges when $r \rightarrow a_{+}$as $1 / \sqrt{r-\bar{a}}$. It has the same singularity structure at $r \rightarrow a_{+}$as the dark matter density because $g^{\prime}(r)=\frac{v_{\text {rot }}^{2}}{r} \times$ $[1+2 f J(r)]$ satisfies Gauss's law. Equation (55) states that, in the limit where all stars are on circular orbits, the star density adopts at $r=a_{+}$the same divergent profile as the dark matter in the caustic surface there. The pileup of circular orbits at $r=a_{+}$is clearly seen in Fig. 3 .

\section{Noncircular orbits}

The observed radial velocity dispersion $\Delta v$ of stars in the Monoceros Ring is of order $20 \mathrm{~km} / \mathrm{s}$. This implies that the stars do not move on circular orbits, but oscillate in the radial direction with typical amplitude

$$
\Delta r \simeq 20 \frac{\mathrm{km}}{\mathrm{s}} \frac{1}{\omega} \simeq 20 \frac{\mathrm{km}}{\mathrm{s}} \frac{1}{\sqrt{2}} \frac{20 \mathrm{kpc}}{v_{\mathrm{rot}}}=1.3 \mathrm{kpc} .
$$

The density profile of stars in the neighborhood of a caustic ring is therefore the profile of Eq. (54) averaged over the length scale $\Delta r$. The sharp features at $r=a$ and $r=a+p$ are then washed out. However, there will be an average relative overdensity within the tricusp

$$
\left.\left\langle\frac{\rho^{\prime}-\rho}{\rho}\right\rangle \simeq a f\left\langle\frac{d J}{d r}\right\rangle\right|_{a<r<a+p} \sim \frac{a f}{p} \simeq 1 \frac{\mathrm{kpc}}{p}
$$

provided $p$ is not much less than $\Delta r$. This is the $100 \%$ average overdensity of ordinary disk stars at the $n=2$ caustic ring of dark matter, announced earlier.

For the proposed interpretation of the Monoceros Ring, the radial velocities acquired by stars as a result of the passing of a caustic ring should be less than the measured radial velocity dispersion of stars in the Monoceros Ring. We now verify that this is the case.

The radial motion of a star in a near circular orbit in the neighborhood of a caustic ring is that of a harmonic oscillator whose equilibrium position is $r^{\prime}(r(l), a(t))$ and whose proper frequency $\omega$ is given by Eq. (40) evaluated at $r^{\prime} . a(t)$ is a smooth function of time. However, as shown in Fig. 3, $r^{\prime}$ as a function of $a$ has kinks at $a=r^{\prime}$ and $a=$ $r^{\prime}-p$. The velocity $\frac{d r^{\prime}}{d t}$ of the equilibrium position changes abruptly when it passes the caustic at those locations. A star in circular orbit in the $z=0$ plane first encounters the caustic at $r^{\prime}=a+p$. For illustrative purposes, we consider the case $a=20 \mathrm{kpc}, t_{0}=13.7 \mathrm{Gyr}, \epsilon=0.3, p=$ $1 \mathrm{kpc}, v=b$, and $f=0.046$. The caustic moves with outward speed

$$
\frac{d a}{d t}=\left(\frac{2}{3}+\frac{2}{9 \epsilon}\right) \frac{a}{t_{0}}=2.0 \mathrm{~km} / \mathrm{s} .
$$

When $r^{\prime}=a+p$, the velocity of the equilibrium position changes abruptly by the amount ( $\delta$ is infinitesimally small)

$$
\begin{aligned}
\Delta \frac{d r^{\prime}}{d t} & =\left[\frac{d r^{\prime}}{d a}\left(r^{\prime}=a+p-\delta\right)-\frac{d r^{\prime}}{d a}\left(r^{\prime}=a+p+\delta\right)\right] \frac{d a}{d t} \\
& \simeq \frac{a f}{2 p+a f} \frac{d a}{d t} \simeq 0.6 \mathrm{~km} / \mathrm{s}
\end{aligned}
$$

Here and below we are neglecting terms of order $f, p / a$, $p / p^{\prime}$, and $a f / p^{\prime}$ versus terms of order one. The proper oscillation frequency after the caustic has passed is

$$
\omega(r=a+p-\delta) \simeq \sqrt{2} \frac{v_{\text {rot }}}{a} \sqrt{1+\frac{a f}{2 p}} \simeq \frac{1}{5 \times 10^{7} \text { years }} .
$$

Thus a star which is in a circular orbit in the $z=0$ plane before the $r^{\prime}=a+p$ caustic passes by, will be oscillating in the radial direction with initial amplitude

$$
A=\frac{1}{\omega} \Delta \frac{d r^{\prime}}{d t} \simeq 30 \mathrm{pc}
$$

after that caustic passes by. Stars not in the $z=0$ plane do not go through the caustic cusp at $r^{\prime}=a+p$, and presumably make smoother transitions through the caustic than the stars in the $z=0$ plane. So, Eq. (61) provides an upper limit for the $z \neq 0$ stars. Stars receive a second jolt when the $r^{\prime}=a$ caustic surface passes by. The analogous quantities for that transition are

$$
\begin{aligned}
\Delta \frac{d r^{\prime}}{d t} & =\left[\frac{d r^{\prime}}{d a}\left(r^{\prime}=a_{-}\right)-\frac{d r^{\prime}}{d a}\left(r^{\prime}=a_{+}\right)\right] \frac{d a}{d t} \\
& \simeq-\frac{d a}{d t} \simeq-2 \mathrm{~km} / \mathrm{s} \\
\omega\left(r=a_{-}\right) & \simeq \sqrt{2} \frac{v_{\text {rot }}}{a} \simeq \frac{1}{6 \times 10^{7} \text { year }} \\
A & =\left|\Delta \frac{d r^{\prime}}{d t}\right| \frac{1}{\omega} \simeq 130 \mathrm{pc} .
\end{aligned}
$$

After the equilibrium position $r^{\prime}$ has passed by the caustic surface at $r^{\prime}=a, r^{\prime}$ decreases very quickly, implying a depletion on the $r<a$ side of the caustic. This depletion is evident in Fig. 3. 


\section{CONCLUSIONS}

The flow of cold collisionless dark matter in and out of the gravitational potential of a galaxy necessarily forms inner and outer caustics. The inner caustics are rings in the galactic plane provided the angular momentum distribution of the infalling dark matter is characterized by net overall rotation. Assuming self-similar infall, the radii of the caustic rings of dark matter in our galaxy were predicted to be $40 \mathrm{kpc} / n$ with $n=1,2,3, \ldots$ Because the Monoceros Ring of stars is located near the second caustic ring of dark matter we looked for processes by which the latter may cause the former.

We have identified two such processes. The first is the flow of gas in the disk towards the sharp angular velocity minimum located at the caustic ring radius, increasing the rate of star formation there. To the extent that this process is responsible for the formation of the Monoceros Ring, the Ring stars are predicted to be bluer than average. The second process is the adiabatic deformation of star orbits in the neighborhood of the caustic ring. As the spatial dependence of the gravitational field of a caustic ring is known, it is straightforward to obtain the map of initial to final orbits for disk stars. The resulting enhancement of disk star density at the location of the second caustic ring is of order $100 \%$. Because of uncertainties in the caustic parameters $\frac{v}{b}$ and $p$, and in the velocity distribution of the disk stars, the strength of the enhancement can only be estimated within a factor of 2 or so.
The self-similar infall model of galactic halo formation is expected to describe the halos of all isolated spiral galaxies. The caustic rings of dark matter in exterior galaxies may also be revealed by the baryonic matter they attract. Our analysis is relevant to those cases as well. It should also help in interpreting the observational evidence for the other caustic rings in the Milky Way. As was mentioned in Sec. IIC, there is evidence for rings $n=3$ and rings $n=5,6,7,8,9,10,11,12,13$, and 14 in the form of sharp rises in the Galactic rotation curve. A triangular feature in the IRAS map of the Galactic disk provides additional evidence for ring $n=5$. In each case, the rings are allegedly revealed by their gravitational effect on the distribution of nearby baryonic matter, which is the central topic of our paper.

The existence of caustics has implications for most approaches to the detection of dark matter, including direct searches of WIMPs [29] and axions [30], the gamma ray signal from WIMP annihilation [31], and gravitational lensing [32].

\section{ACKNOWLEDGMENTS}

We thank Scott Tremaine for extended discussions and for several insights which played a crucial role in the development of the results presented here. This work was supported in part by the U.S. Department of Energy under Grant No. DE-FG02-97ER41209. P. S. gratefully acknowledges the hospitality of the Aspen Center for Physics while he was working on this project.
[1] H. Newberg et al., Astrophys. J. 569, 245 (2002).

[2] B. Yanny et al., Astrophys. J. 588, 824 (2003).

[3] R. A. Ibata et al., Mon. Not. R. Astron. Soc. 340, L21 (2003).

[4] H. J. Rocha-Pinto et al., Astrophys. J. 594, L115 (2003).

[5] J. D. Crane et al., Astrophys. J. 594, L119 (2003).

[6] B. Yanny et al., Astrophys. J. 605, 575 (2004).

[7] A. Helmi et al., Astrophys. J. 592, L35 (2003).

[8] N. F. Martin et al., Mon. Not. R. Astron. Soc. 362, 906 (2005).

[9] J. Peñarrubia et al., Astrophys. J. 626, 128 (2005).

[10] Y. Momany et al., Astron. Astrophys. 421, L29 (2004).

[11] Y. Momany et al., Astron. Astrophys. 451, 515 (2006).

[12] P. Sikivie, Phys. Lett. B 567, 1 (2003).

[13] P. Sikivie, Phys. Lett. B 432, 139 (1998).

[14] P. Sikivie and J. R. Ipser, Phys. Lett. B 291, 288 (1992).

[15] P. Sikivie, Phys. Rev. D 60, 063501 (1999).

[16] S. Tremaine, Mon. Not. R. Astron. Soc. 307, 877 (1999).

[17] A. Natarajan and P. Sikivie, Phys. Rev. D 72, 083513 (2005).

[18] A. Natarajan and P. Sikivie, Phys. Rev. D 73, 023510 (2006).
[19] J. A. Fillmore and P. Goldreich, Astrophys. J. 281, 1 (1984).

[20] E. Bertschinger, Astrophys. J. Suppl. Ser. 58, 39 (1985).

[21] P. Sikivie, I. Tkachev, and Y. Wang, Phys. Rev. Lett. 75, 2911 (1995); Phys. Rev. D 56, 1863 (1997).

[22] W. Kinney and P. Sikivie, Phys. Rev. D 61, 087305 (2000).

[23] A. G. Doroshkevich, Astrofiz. 6, 581 (1970) [Astrophys. 6, 320 (1973)]; P. J.E. Peebles, Astrophys. J. 277, 470 (1984); Y. Hoffmann and J. Shaham, Astrophys. J. 297, 16 (1985).

[24] A. Riess et al., Astron. J. 116, 1009 (1998); S. Perlmutter et al., Astrophys. J. 517, 565 (1999); C. L. Bennett et al., Astrophys. J. Suppl. Ser. 148, 1 (2003).

[25] D. P. Clements, Astrophys. J. 295, 422 (1985).

[26] D. Lynden-Bell and J. E. Pringle, Mon. Not. R. Astron. Soc. 168, 603 (1974); J.E. Pringle, Annu. Rev. Astron. Astrophys. 19, 137 (1981).

[27] N.F. Martin et al., Mon. Not. R. Astron. Soc. Lett. 367, L69 (2006).

[28] J. Binney and S. Tremaine, Galactic Dynamics (Princeton University Press, Princeton, NJ, 1987).

[29] J.D. Vergados, Phys. Rev. D 63, 063511 (2001); A. M. 
Green, Phys. Rev. D 63, 103003 (2001); G. Gelmini and P. Gondolo, Phys. Rev. D 64, 023504 (2001); J. D. Vergados, Phys. Rev. D 67, 103003 (2003); F.-S. Ling, P. Sikivie, and S. Wick, Phys. Rev. D 70, 123503 (2004); Chris Savage, Katherine Freese, and Paolo Gondolo, Phys. Rev. D 74, 043531 (2006); V. Barger et al., Phys. Rev. D 75, 115002 (2007).

[30] L. Duffy et al., Phys. Rev. Lett. 95, 091304 (2005); Phys. Rev. D 74, 012006 (2006).

[31] L. Bergstrom, L. Edsjo, and C. Gunnarsson, Phys. Rev. D
63, 083515 (2001); C. Hogan, Phys. Rev. D 64, 063515 (2001); L. Pieri and E. Branchini, J. Cosmol. Astropart. Phys. 05 (2005) 007; R. Mohayaee and S. Shandarin, Mon. Not. R. Astron. Soc. 366, 1217 (2006); A. Natarajan, Phys. Rev. D 75, 123514 (2007).

[32] C. Hogan, Astrophys. J. 527, 42 (1999); C. Charmousis, V. Onemli, Z. Qiu, and P. Sikivie, Phys. Rev. D 67, 103502 (2003); R. Gavazzi, R. Mohayaee, and B. Fort, Astron. Astrophys. 454, 715 (2006); V. Onemli, Phys. Rev. D 74, 123010 (2006); Int. J. Mod. Phys. D 15, 2059 (2006). 